JOURNAL OF MECHANICS IN MEDICINE AND BIOLOGY

DOI10.1142/S0219519417500130

IN PRESS ${ }^{\mathrm{TH}}$ APRIL 2016

\title{
PERISTALTIC FLOW AND HYDRODYNAMIC DISPERSION OF A REACTIVE MICROPOLAR FLUID- SIMULATION OF CHEMICAL EFFECTS IN THE DIGESTIVE PROCESS
}

\author{
G. Ravi Kiran and G. Radhakrishnamacharya \\ Department of Mathematics, National Institute of Technology, Warangal, India
}

\section{O. Anwar Bég*}

Petroleum and Gas Engineering Division, Spray Research Group, Room G77, Newton Building, School of Computing, Science and Engineering (CSE), University of Salford, M54WT, UK

* Corresponding Author; Email- $\underline{\text { O.A.Beg@salford.ac.uk }}$

\begin{abstract}
:
The hydrodynamic dispersion of a solute in peristaltic flow of a reactive incompressible micropolar biofluid is studied as a model of chyme transport in the human intestinal system with wall effects. The long wavelength approximation, Taylor's limiting condition and dynamic boundary conditions at the flexible walls are used to obtain the average effective dispersion coefficient in the presence of combined homogeneous and heterogeneous chemical reactions. The effects of various pertinent parameters on the effective dispersion coefficient are discussed. It is observed that average effective dispersion coefficient increases with amplitude ratio which implies that dispersion is enhanced in the presence of peristalsis. Furthermore average effective dispersion coefficient is also elevated with the micropolar rheological and wall parameters. Conversely dispersion is found to decrease with cross viscosity coefficient, homogeneous and heterogeneous chemical reaction rates. The present simulations provide an important benchmark for future chemo-fluid-structure interaction computational models.
\end{abstract}

Keywords: Chyme dynamics; Dispersion, Irreversible Chemical Reaction, Peristalsis, Micropolar fluid, Wall effects; Angular velocity; Fluid-structure interaction; Intestine. 


\section{Introduction}

Although frequently neglected in mathematical studies of peristaltic flows in the intestine, chemical reactions [1] bear an important influence on this complex physiological process. At all stages of the digestive journey, reactions take place. These commence with the mastication in the mouth, which triggers production of the enzyme, ptyalin, by the salivary glands, and this serves to chemically break down starches into dextrose and maltose (glucose types) by hydrogenation. Further in the digestive system, chyme is produced which is semi-fluid mass of partly digested food that is expelled from the stomach into the duodenum and migrates via intestines. Chyme is manufactured via the mechanical and chemical breakdown of a bolus and consists of partiallydigested food, water, hydrochloric acid, and various digestive enzymes. The mechanism of propulsion is peristalsis and this involves rhythmic expansions and contractions of the conduit walls which serve to propel chyme slowly. Chyme is a thick creamy liquid and exhibits significant non-Newtonian characteristics [2-4]. Simulation of chyme dynamics in the intestinal tract therefore requires models which can simulate fluid-structure interaction, rheology and chemical reaction (which extracts nutrients also) in addition to hydrodynamics dispersion.

Since the classical work of Taylor [5], the dispersion of a substance that is miscible with a fluid flowing through a tube or channel has been extensively studied, both theoretically and experimentally. Hydrodynamic dispersion [6] is a mechanism that enhances the rate of broadening of a solute cloud in flow through a tube or channel, and can therefore be utilized as an effective means to accomplish dilution or mixing. Dispersion plays a central role in chyme transport and also in other applications as diverse as chromatographic separations in chemical engineering, pollutant transport in the environment, the mixing and transport of drugs or toxins in physiological systems, and so on ( $\mathrm{Ng}$ [7]). Dispersion of a solute in a viscous fluid under 
different conditions has been studied by Aris [8], Padma and Ramana Rao [9], Gupta and Gupta [10], Ramana Rao and Padma [11]. Subsequently, Chandra and Agarwal [12], Philip and Chandra [13], Alemayehu and Radhakrishnamacharya [14, 15] extended the analysis of Taylor to non-Newtonian fluids.

Peristalsis, as elaborated earlier, is known to be one of the main mechanisms for fluid transport in biological systems. From the point of view of fluid mechanics, peristaltic pumping is characterized by dynamic interaction of fluid flow with the movement of flexible boundaries. In this context it falls in the realm of "moving boundary problems" in applied mathematics [16] or "fluid-structure interaction (FSI) problems" in engineering sciences [17]. A number of biomedical instruments including blood pumps in dialysis and the heart lung machine use this principle. Peristaltic pumps are also found in the bio-chemical industry where they provide safer and more efficient transport of toxic wastes. A further application is the roller/finger pump employed to pump slurries and corrosive fluids. Several investigators have analyzed the peristaltic motion of both Newtonian and non-Newtonian fluids in mechanical as well as physiological systems. Representative studies in this regard are Fung and Yih [18], Shehawey and Sebaei [19], Takagi and Balmforth [20], Radhakrishnamacharya [21], Ramachandra Rao and Mishra [22], Tripathi and Bég [23] and Bég et al. [24].

The peristaltic rheological models alluded to in [21-24] have generally used viscoelastic and simpler rheological formulations to represent the shear-stress strain relationships of biophysical suspensions, of which chyme is an example. However it has been established for some time that physiological fluids possess a micro-structure owing to the presence of for example proteins, enzymes, cells and other constituents within the carrier fluid. Micro-continuum fluid dynamics 
was introduced by Eringen in the 1960s [25] to address these aspects and succinctly accounts for the microscopic effects arising from the local structure of micro-motions of the fluid elements.

A special case of Eringen's general micro-morphic fluid model is the micropolar model [26], which restricts the form of the gyration tensor and this robust theory accurately simulates fluids consisting of a suspension of small, rigid elements. These substructure particles can sustain rotary motions (micro-rotation) and support surface and body couples. Micropolar fluid theory [27] effectively accounts for the rotation of fluid particles by means of an independent kinematic vector termed the micro-rotation vector. Micropolar theory has been proven to correlate well with clinical findings, as elaborated for example by Tozeren and Skalak [28] and Bhargava et al. [29]. Furthermore micropolar theory shows great promise in analyzing a wide range of other complex fluids including lubricants, colloidal suspensions, polymeric fluids, liquid crystals and geophysical slurries. Several studies of peristaltic micropolar flows have been communicated in the biomechanics literature. For example, Muthu et al. [30], Sankad et al. [31] have investigated the influence of wall properties on the peristaltic motion of micropolar fluid under different conditions. Abd-Alla et al. [32] have studied the effect of rotation and magnetic field on peristaltic transport of a micropolar fluid through a porous medium.

Motivated by addressing more accurately the physics of chime transport in intestines, the present study analyzes the combined effects of homogeneous and heterogeneous chemical reactions in the peristaltic flow and hydrodynamic dispersion of solute in a micropolar fluid with wall effects. Such a study has thusfar not appeared in the literature. It is envisaged that peristalsis may have significant effects on the dispersion of a solute in the fluid flow and this may lead to better understanding of the actual multi-physical mechanisms inherent to chime dynamics, and indeed other physiological systems. Using the long wavelength approximation 
and Taylor's approach, analytical expressions have been obtained for the average effective dispersion coefficient, in the presence of combined homogeneous and heterogeneous irreversible chemical reactions. Mathematica software has been employed to compute solutions in order to study the effects of various biophysical and geometric parameters on flow characteristics.

\section{Mathematical Model}

Consider the hydrodynamic dispersion of a solute in peristaltic flow of a micropolar fluid (chyme) in an infinite uniform channel of width $2 d$ and with flexible walls on which are imposed traveling sinusoidal waves of long wavelength. A Cartesian coordinate system $(x, y)$ is chosen wherein the $x$-axis aligned with the center line of the channel. The traveling waves are represented by the relationship:

$$
y= \pm h= \pm\left[d+a \sin \frac{2 \pi}{\lambda}(x-c t)\right]
$$

where $a$ is the amplitude, $c$ is the speed and $\lambda$ is the wavelength of the peristaltic wave. The regime studied is illustrated in Fig.1. The governing equation of motion of the flexible wall may be expressed as (Mittra and Prasad [33])

$$
L(h)=p-p_{0}
$$

where $L$ is an operator which is used to represent the motion of stretched membrane with damping forces such that

$$
L=-T \frac{\partial^{2}}{\partial x^{2}}+m \frac{\partial^{2}}{\partial t^{2}}+\mathrm{C} \frac{\partial}{\partial t}
$$

Here $T$ is the tension in the membrane, $m$ is the mass per unit area and $\mathrm{C}$ is the coefficient of viscous damping force. This approach simulates the deformability of the conduit wall allowing for fluid-structure interaction in the peristaltic flow. 
The governing equations for mass and momentum conservation for two-dimensional, unsteady flow of an incompressible micropolar fluid for the present regime, take the form (Muthu et al. [34]) :

$$
\begin{aligned}
& \frac{\partial u}{\partial x}+\frac{\partial v}{\partial y}=0 \\
& \rho\left[\frac{\partial u}{\partial t}+u \frac{\partial u}{\partial x}+v \frac{\partial u}{\partial y}\right]=-\frac{\partial p}{\partial x}+\left(\frac{2 \mu+\kappa}{2}\right)\left[\frac{\partial^{2} u}{\partial x^{2}}+\frac{\partial^{2} u}{\partial y^{2}}\right]+\kappa \frac{\partial g}{\partial y} \\
& \rho\left[\frac{\partial v}{\partial t}+u \frac{\partial v}{\partial x}+v \frac{\partial v}{\partial y}\right]=-\frac{\partial p}{\partial y}+\left(\frac{2 \mu+\kappa}{2}\right)\left[\frac{\partial^{2} v}{\partial x^{2}}+\frac{\partial^{2} v}{\partial y^{2}}\right]-\kappa \frac{\partial g}{\partial x} \\
& \rho J\left[\frac{\partial g}{\partial t}+u \frac{\partial g}{\partial x}+v \frac{\partial g}{\partial y}\right]=-2 \kappa g+\gamma\left[\frac{\partial^{2} g}{\partial x^{2}}+\frac{\partial^{2} g}{\partial y^{2}}\right]+\kappa\left[\frac{\partial v}{\partial x}-\frac{\partial u}{\partial y}\right]
\end{aligned}
$$

where $u$ and $v$ are the velocity components in the $\mathrm{x}$ and $\mathrm{y}$ directions respectively, $g$ is the microrotation component, $\rho$ is the density, $p$ is the pressure, $J$ is the microinertia constant, $\mu$ is the coefficient of viscosity, $\kappa$ and $\gamma$ are the viscosity coefficients for the micropolar fluid. Under the long wavelength approximation and neglecting inertial effects (which are dominated by viscous effects), the governing equations for the present problem reduce to,

$$
\begin{aligned}
& \frac{\partial u}{\partial x}+\frac{\partial v}{\partial y}=0 \\
& -\frac{\partial p}{\partial x}+\left(\frac{2 \mu+\kappa}{2}\right) \frac{\partial^{2} u}{\partial y^{2}}+\kappa \frac{\partial g}{\partial y}=0 \\
& -\frac{\partial p}{\partial y}=0 \\
& -2 \kappa g+\gamma \frac{\partial^{2} g}{\partial y^{2}}-\kappa \frac{\partial u}{\partial y}=0
\end{aligned}
$$


It is assumed that $p_{0}=0$ and the channel walls are inextensible so that only lateral motion takes place and the horizontal displacement of the wall is zero. The no-slip boundary conditions for the velocity and microrotation are given by

$$
u=0, g=0 \text { at } y= \pm h
$$

The dynamic boundary conditions at the flexible walls (Mittra and Prasad [33]) can be written as:

$$
\begin{aligned}
& \frac{\partial}{\partial x} L(h)=\left(\frac{2 \mu+\kappa}{2}\right) \frac{\partial^{2} u}{\partial y^{2}}+\kappa \frac{\partial g}{\partial y} \quad \text { at } \quad y= \pm h \\
& \text { where } \frac{\partial}{\partial x} L(h)=\frac{\partial p}{\partial x}=-T \frac{\partial^{3} h}{\partial x^{3}}+m \frac{\partial^{3} h}{\partial x \partial t^{2}}+\mathrm{C} \frac{\partial^{2} h}{\partial x \partial t}
\end{aligned}
$$

Solving equations (9) to (11) under the boundary conditions (12) and (13), we get

$$
u(y)=-\frac{P^{\prime}}{2 \mu}\left[\left(h^{2}-y^{2}\right)+\frac{2 \kappa h}{b(2 \mu+\kappa)} \frac{1}{\sinh (b h)}(\cosh (b y)-\cosh (b h))\right]
$$

where $P^{\prime}=-T \frac{\partial^{3} h}{\partial x^{3}}+m \frac{\partial^{3} h}{\partial x \partial t^{2}}+C \frac{\partial^{2} h}{\partial x \partial t}$ and $b=\sqrt{\frac{4 \mu \kappa}{\gamma(2 \mu+\kappa)}}$

Further, the mean velocity is defined as

$$
\bar{u}=\frac{1}{2 h} \int_{-h}^{h} u(y) d y
$$

Substituting equation (14) in equation (16), we get

$$
\bar{u}=-\frac{P^{\prime}}{\mu}\left[\frac{h^{2}}{3}+\frac{\kappa}{b(2 \mu+\kappa)}\left(\frac{1}{b}-\frac{h \cosh (b h)}{\sinh (b h)}\right)\right]
$$


If we now consider convection across a plane moving with the mean speed of the flow, then relative to this plane, the fluid velocity is given by (Gupta and Gupta [10], Alemayehu and Radhakrishnamacharya $[14,15])$

$$
u_{x}=u-\bar{u}
$$

Substituting equations (14) and (17) in equation (18), we arrive at:

$$
u_{x}=-\frac{P^{\prime}}{\mu}\left[\left(\frac{h^{2}}{6}-\frac{y^{2}}{2}\right)+\frac{\kappa}{b(2 \mu+\kappa)}\left(\frac{h \cosh (b y)}{\sinh (b h)}-\frac{1}{b}\right)\right]
$$

\subsection{Diffusion with Combined Homogeneous and Heterogeneous Chemical Reactions}

Following Taylor [5] and Gupta and Gupta [10], the equation for the concentration $C$ of the solute (representing for example gastric juice in chyme) for the present problem under isothermal conditions is given by:

$$
\frac{\partial C}{\partial t}+u \frac{\partial C}{\partial x}=D \frac{\partial^{2} C}{\partial y^{2}}-k_{1} C
$$

where $D$ is the molecular diffusion coefficient (species diffusivity) and $k_{1}$ is the first order reaction rate constant. For typical values of physiologically relevant parameters of this problem, it is assumed that $\bar{u}=c$ (Alemayehu and Radhakrishnamacharya [14, 15]). Using this condition, it is pertinent to make use of the following dimensionless quantities,

$$
\theta=\frac{t}{\bar{t}}, \quad \bar{t}=\frac{\lambda}{\bar{u}}, \quad \eta=\frac{y}{d}, \quad \xi=\frac{x-\bar{u} t}{\lambda}, \quad H=\frac{h}{d}, \quad P=\frac{d^{2}}{\mu c} P^{\prime}
$$

Equation (15) thereby reduces to :

$$
P=-\varepsilon\left[\left(E_{1}+E_{2}\right)(2 \pi)^{3} \cos (2 \pi \xi)-E_{3}(2 \pi)^{2} \sin (2 \pi \xi)\right] .
$$


Here $\varepsilon\left(=\frac{a}{d}\right)$ is the amplitude ratio, $E_{1}\left(=-\frac{T d^{3}}{\lambda^{3} \mu c}\right)$ is the wall rigidity, $E_{2}\left(=\frac{m c d^{3}}{\lambda^{3} \mu}\right)$ is the stiffness and $E_{3}\left(=\frac{C d^{3}}{\mu \lambda^{2}}\right)$ is the viscous damping force in the wall.

Further, equations (19) and (20) reduce to

$$
\begin{aligned}
& u_{x}=\left(-\frac{d^{2}}{\mu} P\right)\left[\frac{1}{2}\left(\frac{H^{2}}{3}-\eta^{2}\right)+\frac{N}{M}\left(\frac{H \cosh (M N \eta)}{\sinh (M N H)}-\frac{1}{M N}\right)\right] \\
& \frac{\partial^{2} C}{\partial \eta^{2}}-\frac{k_{1} d^{2}}{D} C=\frac{d^{2}}{\lambda D} u_{x} \frac{\partial C}{\partial \xi}
\end{aligned}
$$

where $M=2 d\left(\frac{\mu}{\gamma}\right)^{1 / 2}$ is the micropolar parameter, $N=\left(\frac{\mu_{1}}{2+\mu_{1}}\right)^{1 / 2}$ is the coupling parameter, $\mu_{1}=\frac{\kappa}{\mu}$ is the cross viscosity coefficient and $b=\frac{M N}{d}$. It is assumed that a first order irreversible chemical reaction takes place both in the bulk of the fluid (homogeneous) as well as at the walls (heterogeneous) of the channel which are assumed to be catalytic to chemical reaction. Thus, the corresponding boundary conditions at the walls (Philip and Chandra [13]) are given by

$$
\begin{aligned}
& \frac{\partial C}{\partial y}+f C=0 \text { at } y=h=\left[d+a \sin \frac{2 \pi}{\lambda}(x-c t)\right] \\
& \frac{\partial C}{\partial y}-f C=0 \text { at } y=-h=-\left[d+a \sin \frac{2 \pi}{\lambda}(x-c t)\right]
\end{aligned}
$$

If we introduce the dimensionless variables (21), the boundary conditions become

$$
\begin{aligned}
& \frac{\partial C}{\partial \eta}+\beta C=0 \text { at } \eta=H=[1+\varepsilon \sin (2 \pi \xi)] \\
& \frac{\partial C}{\partial \eta}-\beta C=0 \text { at } \eta=-H=-[1+\varepsilon \sin (2 \pi \xi)]
\end{aligned}
$$


where $\beta=f d$ is the heterogeneous reaction rate parameter corresponding to catalytic reaction at the walls.

The solution of the equation (24) under the boundary conditions (27) and (28) is

$$
\begin{aligned}
C(\eta)=\left(\frac{-d^{4}}{\lambda \mu D} \frac{\partial C}{\partial \xi} P\right)[ & -\frac{A}{L} \cosh (\alpha \eta)-\frac{1}{2 \alpha^{2}}\left(\frac{H^{2}}{3}-\frac{2}{\alpha^{2}}-\eta^{2}\right)+ \\
& \left.\frac{N}{M}\left(\frac{H}{M^{2} N^{2}-\alpha^{2}} \frac{\cosh (M N \eta)}{\sinh (M N H)}+\frac{1}{M N \alpha^{2}}\right)\right]
\end{aligned}
$$

where $A=\left[\frac{H}{\alpha^{2}}+\frac{N^{2} H}{M^{2} N^{2}-\alpha^{2}}+\frac{\beta}{\alpha^{2}}\left(\frac{H^{2}}{3}+\frac{1}{\alpha^{2}}\right)+\frac{\beta N}{M}\left(\frac{H}{M^{2} N^{2}-\alpha^{2}} \operatorname{coth}(M N H)+\frac{1}{M N \alpha^{2}}\right)\right]$

and $\alpha=\left(\frac{k_{1} d^{2}}{D}\right)^{1 / 2}$

The volumetric rate $Q$ at which the solute is transported across a section of the channel of unit breadth is defined by:

$$
Q=\int_{-H}^{H} C u_{x} d \eta
$$

Substituting (23) and (29) in (30), we get the volumetric rate $Q$ as:

$$
Q=-2 \frac{d^{6}}{\lambda \mu^{2} D} \frac{\partial C}{\partial \xi} G\left(\xi, \varepsilon, \alpha, \beta, E_{1}, E_{2}, E_{3}, \mu_{1}, M\right)
$$

where 
$G\left(\xi, \varepsilon, \alpha, \beta, E_{1}, E_{2}, E_{3}, \mu_{1}, M\right)=$

$$
\begin{aligned}
& \left(\frac{-P^{2}}{2}\right)\left[2 H\left(l_{1} l_{4}\right)+\left(l_{1} l_{5}+l_{2} l_{4}\right) \frac{2 H^{3}}{3}+\left(l_{1} l_{6}\right) \frac{2 \sinh (\alpha H)}{\alpha}+\left(l_{1} l_{7}+l_{3} l_{4}\right) \frac{2 \sinh (M N H)}{M N}+\right. \\
& \left(l_{2} l_{5}\right)\left(\frac{2 H^{5}}{5}\right)+\left(l_{3} l_{6}\right)\left(\frac{2}{M^{2} N^{2}-\alpha^{2}}\right)[M N \cosh (\alpha H) \sinh (M N H)-\alpha \cosh (M N H) \sinh (\alpha H)]+ \\
& \left(l_{3} l_{7}\right)\left(H+\frac{\sinh (2 M N H)}{2 M N}\right)+\left(\frac{l_{2} l_{6}}{\alpha^{3}}\right)\left[-4 \alpha H \cosh (\alpha H)+2\left(2+\alpha^{2} H^{2}\right) \sinh (\alpha H)\right]+ \\
& \left.\left(l_{3} l_{5}+l_{2} l_{7}\right) \frac{1}{M^{3} N^{3}}\left[-4 M N H \cosh (M N H)+2\left(2+M^{2} N^{2} H^{2}\right) \sinh (M N H)\right]\right]
\end{aligned}
$$

where

$$
\begin{aligned}
& l_{1}=\frac{H^{2}}{6}-\frac{1}{M^{2}}, \quad l_{2}=\frac{-1}{2}, \quad l_{3}=\frac{H N}{M \sinh (M N H)}, \quad l_{4}=\frac{-1}{\alpha^{2}}\left(\frac{H^{2}}{6}-\frac{1}{\alpha^{2}}-\frac{1}{M N}\right), \\
& l_{5}=\frac{1}{2 \alpha^{2}}, \quad l_{6}=\frac{-A}{L} \quad \text { and } \quad l_{7}=\frac{N}{M} \frac{H}{M^{2} N^{2}-\alpha^{2}} \frac{1}{\sinh (M N H)}
\end{aligned}
$$

Now comparing the equation (31) with Fick's first law of diffusion, the effective dispersion coefficient $D^{*}$ with which the solute disperses relative to a plane moving with the mean speed of the flow, is obtained as,

$$
D^{*}=2 \frac{d^{6}}{\mu^{2} D} G\left(\xi, \varepsilon, \alpha, \beta, E_{1}, E_{2}, E_{3}, \mu_{1}, M\right)
$$

Let the average of $G$ be $\bar{G}$, which is defined by

$$
\bar{G}=\int_{0}^{1} G\left(\xi, \varepsilon, \alpha, \beta, E_{1}, E_{2}, E_{3}, \mu_{1}, M\right) d \xi
$$

\section{Numerical Results and Discussion}

Equation (34) gives the equivalent dispersion coefficient $D^{*}$ through the function $\bar{G}$, which has been computed numerically using MATHEMATICA software and the results are presented 
graphically. The key dimensionless quantities emerging are: the amplitude ratio $\varepsilon$, the homogeneous reaction rate $\alpha$, the heterogeneous reaction rate $\beta$, the cross viscosity coefficient $\mu_{1}$, the micropolar parameter $M$ and the wall (fluid-structure interaction) parameters $E_{1}, E_{2}, E_{3}$. Further, from the equations (13) and (22) we may note that $E_{1}, E_{2}$ and $E_{3}$ cannot be taken as zero simultaneously.

The effect of the rigidity parameter $\left(E_{1}\right)$ on the effective dispersion coefficient is shown in Figs. 2 - 4. It is observed that the dispersion increases with the rigidity parameter in the cases of (i) no stiffness in the wall $\left(E_{2}=0\right)$ and a perfectly elastic channel wall ( $E_{3}=0$ ) (Fig. 2); (ii) stiffness in the wall $\left(E_{2} \neq 0\right)$ and perfectly elastic wall ( $E_{3}=0$ ) (Fig. 3); (iii) no stiffness in the wall $\left(E_{2}=0\right)$ and dissipative wall $\left(E_{3} \neq 0\right)$ (Fig. 4). However the horizontal axes on these graphs represent a different parameter. With increasing rigidity parameter in the case of (i) no stiffness in the wall ( $E_{2}=0$ ) i.e. fig. 2 , the dispersion coefficient values are plateau-profiles and remain at consistent distances from one another, as the heterogeneous reaction rate $\beta$ parameter increases from unity to 10 . In fig. 3 , the $\mathrm{x}$-axis represents amplitude ratio $\varepsilon$, and profiles ascend monotonically for any value of rigidity parameter, commencing at zero for minimum amplitude ratio (origin) and peaking at the maximum value of amplitude ratio.

A nonlinear and positive influence of amplitude ratio on dispersion coefficient is therefore evident in fig. 3. In fig. 4, dispersion coefficient, $\bar{G}$ clearly descends as the homogeneous reaction rate, $\alpha$, increases, demonstrating an inverse relationship. The rate of descent of the profiles is accentuated with greater values of the rigidity parameter $\left(E_{1}\right)$. Maximum dispersion coefficient corresponds to the minimum homogenous reaction rate and the maximum rigidity parameter scenario. This clearly demonstrates that both parameters exert a non-trivial effect on 
hydrodynamic dispersion in peristaltic flow and has implications for the effectiveness of chyme dispersion phenomena [35].

Figs. 5 - 7 show that the dispersion coefficient increases as the stiffness in the wall $\left(E_{2}\right)$ increases for both the cases of perfectly elastic wall $\left(E_{3}=0\right)$ (Fig. 5) and dissipative wall $\left(E_{3} \neq 0\right)$ (Figs. 6 and 7). Dispersion coefficient values are largely invariant with alteration in the heterogeneous chemical reaction rate $\beta$ (fig. 5). The response to a change in amplitude ratio (fig. $6,7)$ and homogeneous reaction rate, is similar to that observed in figs. 3, 4- namely dispersion coefficient sharply ascends with an increase in the former, whereas it decays approximately linearly with a rise in the latter.

Figs. 8 - 10 demonstrate that the average effective dispersion coefficient increases with viscous damping force $\left(E_{3}\right)$. Distinct from earlier graphs, however we observe that a sharp monotonic decay in hydrodynamic dispersion coefficient (fig. 8) accompanies an increase in heterogenous chemical reaction rate $\beta$ whereas in figs 2,5 the profiles remain largely constant with greater values of $\beta$. Fig. 9 illustrates that significantly greater magnitudes of dispersion coefficient are achieved with an increase in amplitude ratio as viscous damping force parameter ( $\left.E_{3}\right)$ is increased, compared with figs. 3 and 6 where rigidity parameter $\left(E_{1}\right)$ and wall stiffness are increased $\left(E_{2}\right)$. Fig. 10 indicates that the effect of greater homogeneous reaction rate, $\alpha$, is also less pronounced less for any value of $E_{3}$, compared with distributions in figs. 4 and 7 , although the over-riding effect is that of depleting hydrodynamic dispersion coefficient.

Figs. 11 and 12 reveal that the effective dispersion coefficient $\bar{G}$ increases with micropolar parameter $M$ whereas it decreases with cross viscosity coefficient $\mu_{1}$ (Figs. 13 and 14). These are true for the cases of (i) stiffness in the wall $\left(E_{2} \neq 0\right)$ and perfectly elastic wall $\left(E_{3}=0\right)$ (Figs. 11 
and 13); (ii) no stiffness in the wall $\left(E_{2}=0\right)$ and dissipative wall $\left(E_{3} \neq 0\right)$ (Figs. 12 and 14) but the variation in case (ii) is not significant. With greater values of $M$, the gyration viscosity is lessened and spin of micro-elements in the biofluid is enhanced. This promotes hydrodynamic dispersion and elevates values of $\bar{G}$.

Figs. 3, 6, 9, 12 and 14 show that the average effective dispersion coefficient increases with amplitude ratio $\varepsilon$. This implies that peristalsis enhances dispersion of a solute in fluid flow. This result agrees with that of Alemayehu and Radhakrishnamacharya [14, 15]. Furthermore, as elaborated earlier, the dispersion coefficient decreases with homogeneous chemical reaction rate parameter $\alpha$ (Figs. 4, 7 and 10) and heterogeneous chemical reaction rate $\beta$ (Figs. 2, 5, 8, 11 and 13), whereas dispersion decreasing with $\beta$ is less significant. This result agrees with that of Padma and Ramana Rao [9], Gupta and Gupta [10], Ramana Rao and Padma [11]. This result is expected since increase in $\alpha$ leads to an increase in number of moles of solute undergoing chemical reaction, and this effectively counter-acts hydrodynamic dispersion.

\section{Conclusions}

The effect of combined homogeneous and heterogeneous chemical reactions on peristaltic flow and hydrodynamic dispersion in a micropolar biofluid with wall effects has been studied analytically under the long wavelength approximation and Taylor's limiting condition. The model developed is relevant to chyme transport in the human digestive system. Mathematica software computations have shown that peristaltic motion enhances dispersion and hydrodynamic dispersion decreases with micropolar cross viscosity coefficient. It is also observed that the effective dispersion coefficient increases with micropolar parameter and the wall parameters i.e. rigidity, stiffness, and viscous damping. Furthermore, it is found that 
average dispersion coefficient decreases with increasing homogeneous and heterogeneous reaction rates. The present analytical study should serve to provide a useful benchmark for more sophisticated 3-D FSI chemo-peristaltic flow simulations with multi-physics software.

\section{References}

[1] D.S. Robertson, The chemical reactions in the human stomach and the relationship to metabolic disorders, Medical Hypotheses, 64, 1127-1131 (2005).

[2] M. Taghipoor, P. Lescoat, J.-R. Licois, C. Georgelin, G. Barles, Mathematical modeling of transport and degradation of feedstuffs in the small intestine, J. Theoretical Biology, 294, 114$121(2012)$.

[3] A. Tharakan, I. T. Norton, P. J. Fryer, S. Bakalis, Mass transfer and nutrient absorption in a simulated model of small intestine, J. Food Science, 75, E339-E346 (2010).

[4] B. R. Stoll, R. P. Batycky, H. R. Leipold, S. Milstein, D. A. Edwards, A theory of molecular absorption from the small intestine, Chemical Engineering Science, 55, 473-489 (2000).

[5] G.I. Taylor, Dispersion of soluble matter in solvent flowing slowly through a tube, Proc. Roy. Soc. Lond., A, 219, 186 - 203 (1953).

[6] D.A. Edwards, H. Brenner and D.T. Wasan, Interfacial Transport Processes and Rheology, Butterworths, USA (1991).

[7] C.O. Ng, Dispersion in steady and oscillatory flows through a tube with reversible and irreversible wall reactions, Proc. Roy. Soc. Lond., A, 462, 481 - 515 (2006).

[8] R. Aris, On the dispersion of a solute in a fluid flowing through a tube, Proc. Roy. Soc. Lond., A, 235, 67 - 77 (1956). 
[9] D. Padma and V.V. Ramana Rao, Effect of homogeneous and heterogeneous reactions on the dispersion of a solute in laminar flow between two parallel porous plates, Indian J. Technol., 14, $410-412(1976)$.

[10] P.S. Gupta and A.S. Gupta, Effect of homogeneous and heterogeneous reactions on the dispersion of a solute in the laminar flow between two plates, Proc. Roy. Soc. Lond., A, 330, 59 -63 (1972).

[11] V.V. Ramana Rao and D. Padma, Homogeneous and heterogeneous reactions on the dispersion of a solute in MHD Couette flow, Curr. Sci., 44, 803 - 804 (1975).

[12] P. Chandra and R.P. Agarwal, Dispersion in simple microfluid flows, Int. J. Eng. Sci., 21, $431-442(1983)$.

[13] D. Philip and P. Chandra, Effects of heterogeneous and homogeneous reactions on the dispersion of a solute in simple micro fluid, Indian J. Pure Appl. Math., 24, 551 - 561 (1993).

[14] H. Alemayehu and G. Radhakrishnamacharya, The dispersion on peristaltic flow of micropolar fluid in a porous medium, J. Porous Media, 15, 1067 - 1077 (2012).

[15] H. Alemayehu and G. Radhakrishnamacharya, Dispersion of a solute in peristaltic motion of a couple stress fluid through a porous medium, Tamkang J. Math., 43, 541 - 555 (2012).

[16] D. Tang andM. C. Shen, Nonstationary peristaltic transport of a heat-conducting fluid, $J$. Mathematical Analysis and Applications, 174, 265-289 (1993).

[17] T.D. Brown and Hung, T. K., Computational and experimental investigations of twodimensional nonlinear peristaltic flows, J. Fluid Mechanics 83, 249-272 (1977)

[18] Y.C. Fung and C.S. Yih, Peristaltic transport, J. Appl. Mech. ,Trans. ASME, 35, $669-675$ (1968). 
[19] E.F.El. Shehawey and W.El. Sebaei, Peristaltic transport in a cylindrical tube through a porous medium, Int. J. Math. Math. Sci., 24, 217 - 230 (2000).

[20] D. Takagi and N.J. Balmforth, Peristaltic pumping of viscous fluid in an elastic tube, $J$. Fluid Mech., 672, 196 - 218 (2011).

[21] G. Radhakrishnamacharya, Long wave length approximation to peristaltic motion of a power-law fluid, Rheol. Acta, 21, 30 - 35 (1982).

[22] A. Ramachandra Rao and M. Mishra, Peristaltic transport of a power-law fluid in a porous tube, J. Non-Newtonian Fluid Mech., 121, 163 - 174 (2004).

[23] D. Tripathi and O. Anwar Bég, A study on peristaltic flow of nanofluids: Application in drug delivery systems, Int. J. Heat Mass Trans., vol. 70, pp. 61 - 70, 2014.

[24] O. Anwar Bég and M.M. Rashidi, Multi-Step DTM simulation of magneto-peristaltic flow of a conducting Williamson viscoelastic fluid, Int. J. Applied Mathematics Mechanics, 9, 22-40 (2013).

[25] A.C. Eringen, Simple microfluids, Int. J. Engineering Science, 2, 205-217 (1964).

[26] A.C. Eringen, Theory of micropolar fluids, J. Math. Mech., 16, 1-18 (1966).

[27] O. Anwar Bég, R. Bhargava and M.M. Rashidi, Numerical Simulation in Micropolar Fluid Dynamics, 244 pages, Lambert Academic, Saarbrucken, Germany (2011).

[28] A. Tözeren and R. Skalak, Micropolar fluids as models for suspensions of rigid spheres, Int. J. Engineering Science, 15, 511-523 (1977).

[29] R. Bhargava, S. Sharma, O. Anwar Bég and Zueco, J, Finite element study of nonlinear two-dimensional deoxygenated biomagnetic micropolar flow, Communications in Nonlinear Science and Numerical Simulation, 15, 1210-1233 (2010). 
[30] P. Muthu, B.V. Ratish Kumar and P. Chandra, Peristaltic motion of micropolar fluid in circular cylindrical tubes: Effect of wall properties, Appl. Math. Model., 32, 2019 - 2033 (2008). [31] G.C. Sankad, G. Radhakrishnamacharya and J.V. Ramana Murthy, Long wavelength approximation to peristaltic motion of micropolar fluid with wall effects. Adv. Appl. Math. Mech., 2, $222-237$ (2010).

[32] A.M. Abd-Alla, S.M. Abo-Dahab and R.D. Al-Simery, Effect of rotation on peristaltic flow of a micropolar fluid through a porous medium with an external magnetic field, J. Magn. Magn. Mater., 348, 33 - 43 (2013).

[33] T.K. Mittra and S.N. Prasad, On the influence of wall properties and Poiseuille flow in peristalsis, J. Biomech., 6, $681-693$ (1973).

[34] P.Muthu, B.V. Ratish Kumar and P. Chandra, On the influence of the wall properties in the peristaltic motion of micropolar fluid, ANZIAM J., 45, 246 - 260 (2003).

[35] R. Roy, F. Rios and D. Riahi, Mathematical models for flow of chyme during gastrointestinal endoscopy, Applied Mathematics, 2, 600-607 (2011). 


\section{FIGURES}

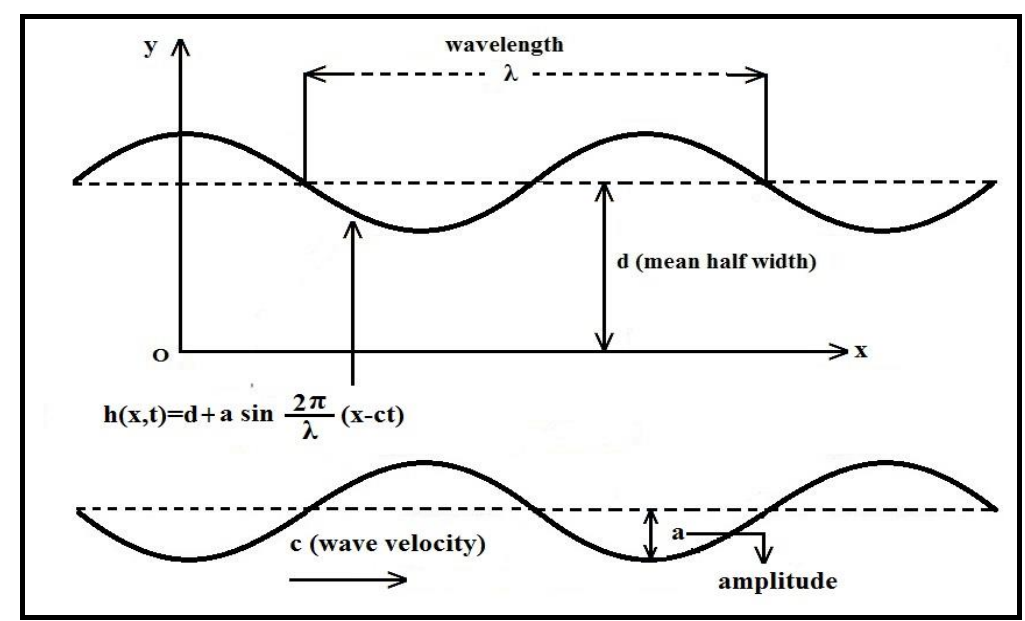

Fig. 1: Geometry of chyme dynamics in an idealized intestinal geometry

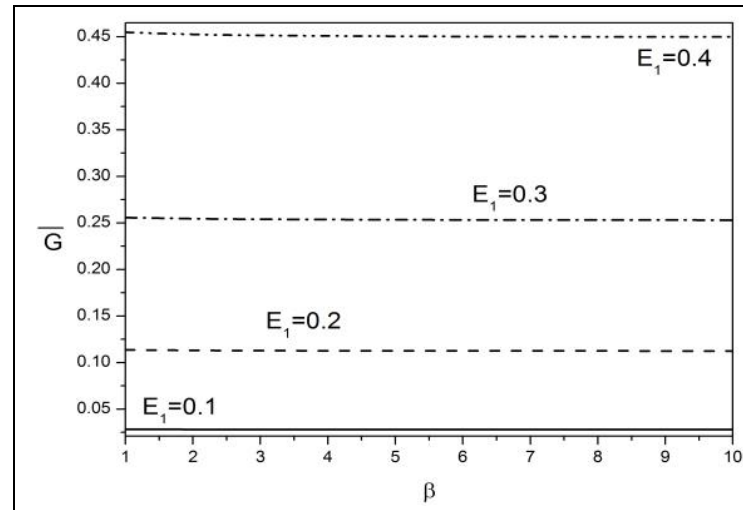

Fig. 2 : Effect of $E_{l}$ on $\bar{G}$

$\left(\varepsilon=0.2, \alpha=0.5, E_{2}=0.0, E_{3}=0.0\right.$, $\mu_{1}=0.04, M=10$ )

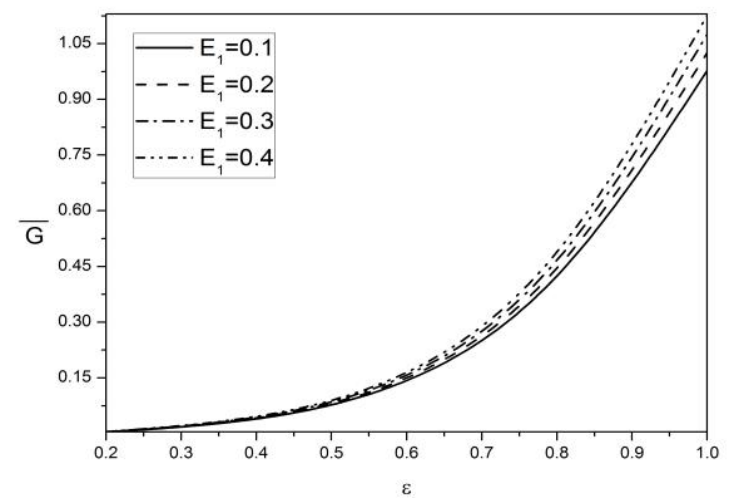

Fig. 3 : Effect of $E_{l}$ on $\bar{G}$ $\left(\alpha=0.5, \beta=5, E_{2}=4.0, E_{3}=0.0\right.$, $\mu_{1}=0.04, M=10$ ) 


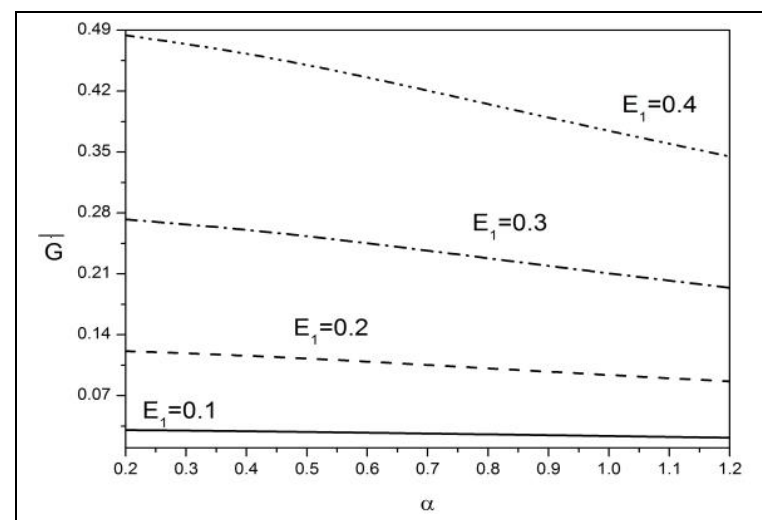

Fig. 4 : Effect of $E_{l}$ on $\bar{G}$ ( $\varepsilon=0.2, \beta=5, E_{2}=0.0, E_{3}=0.06$,

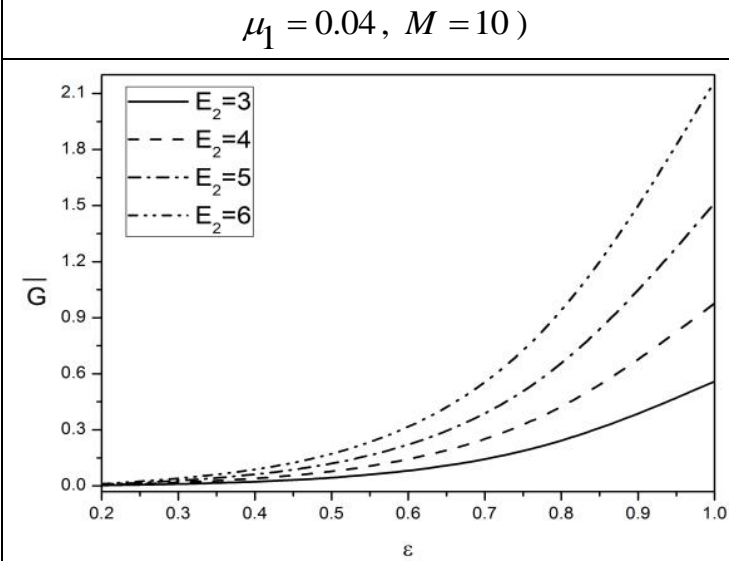

Fig. 6 : Effect of $E_{2}$ on $\bar{G}$

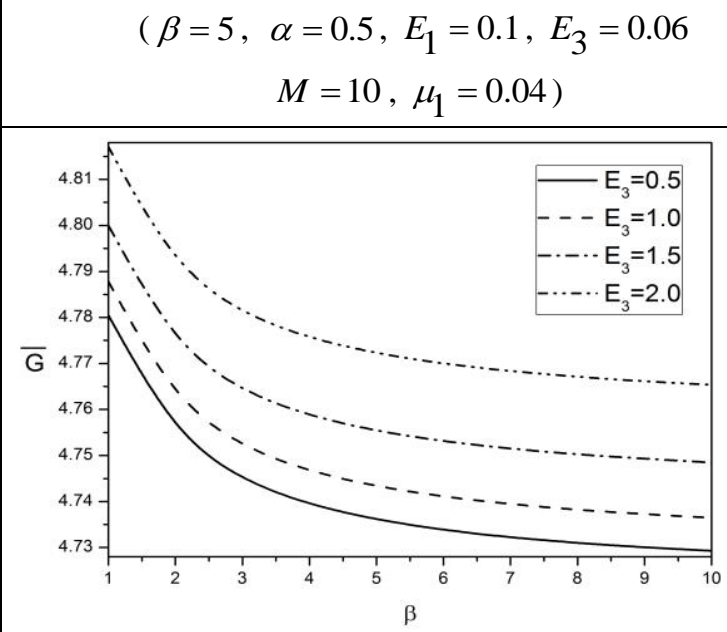

Fig. $8:$ Effect of $E_{3}$ on $\bar{G}$ ( $\varepsilon=0.2, \alpha=0.5, E_{1}=0.1, E_{2}=4.0$, $M=10, \mu_{1}=0.04$ )

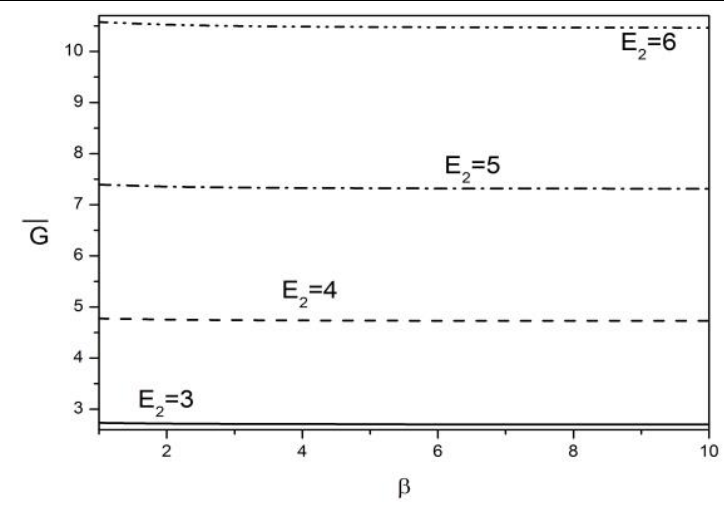

Fig. 5 : Effect of $E_{2}$ on $\bar{G}$ ( $\varepsilon=0.2, \alpha=0.5, E_{1}=0.1, E_{3}=0.0$, $\left.\mu_{1}=0.04, M=10\right)$

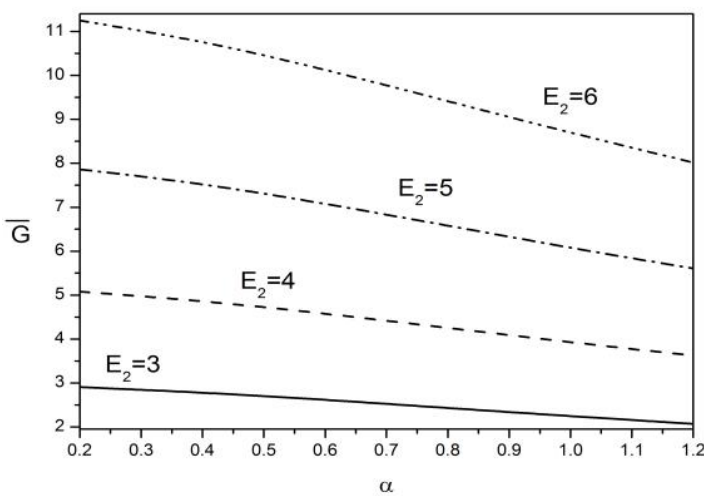

Fig. 7 : Effect of $E_{2}$ on $\bar{G}$ ( $\varepsilon=0.2, \beta=5, E_{1}=0.1, E_{3}=0.06$, $M=10, \mu_{1}=0.04$ )

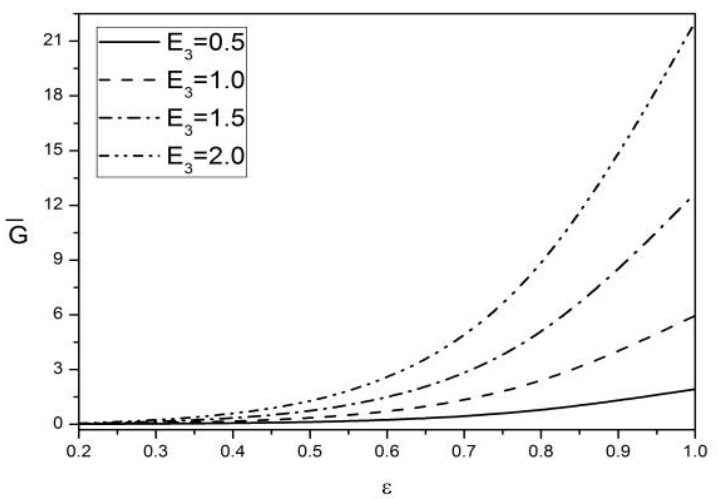

Fig. 9 : Effect of $E_{3}$ on $\bar{G}$ $\left(\beta=5, \alpha=0.5, E_{1}=0.1, E_{2}=0.0\right.$, $\left.M=10, \mu_{1}=0.04\right)$ 


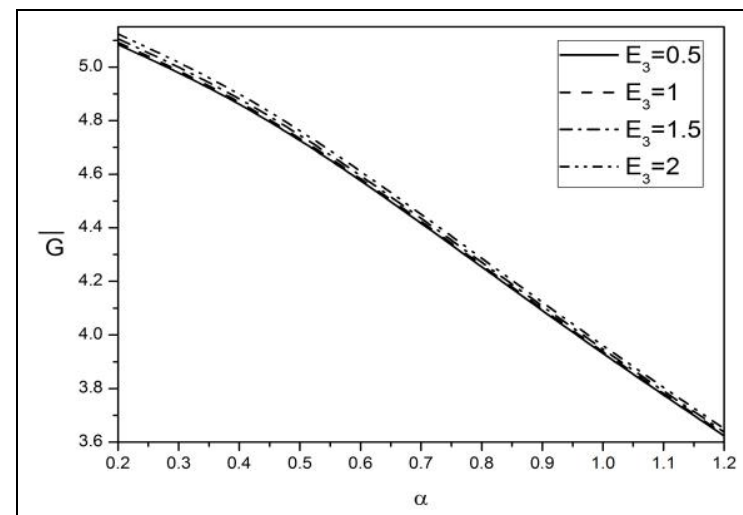

Fig. 10 : Effect of $E_{3}$ on $\bar{G}$ $\left(\varepsilon=0.2, \beta=5, E_{1}=0.1, E_{2}=4.0\right.$, $M=10, \mu_{1}=0.04$ )

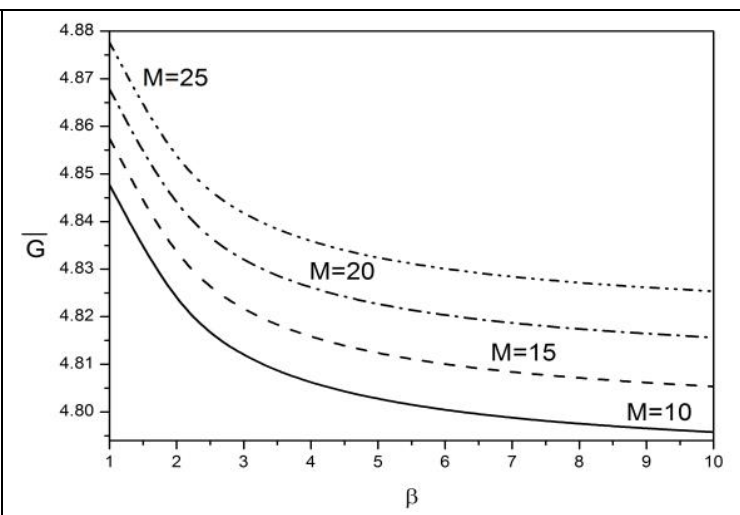

Fig. 11 : Effect of $\mathrm{M}$ on $\bar{G}$ $\left(\varepsilon=0.2, \alpha=0.5, E_{1}=0.1, E_{2}=4.0\right.$, $E_{3}=0.0, \mu_{1}=0.02$ )

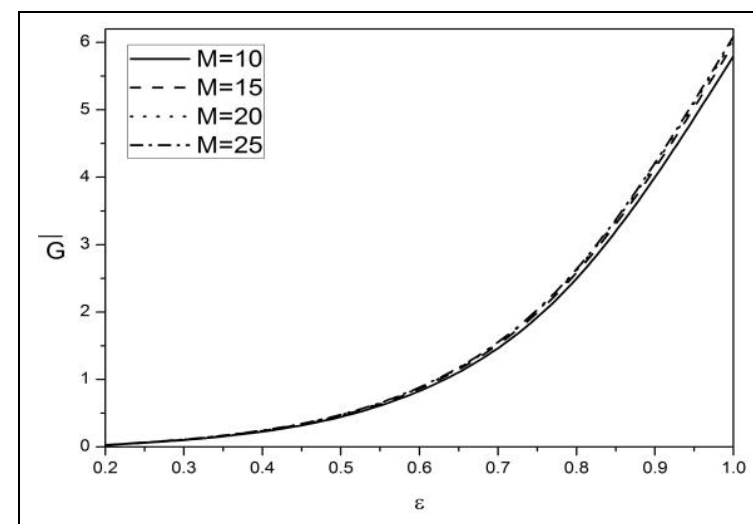

Fig. 12 : Effect of $\mathrm{M}$ on $\bar{G}$ $\left(\beta=5, \alpha=0.5, E_{1}=0.1, E_{2}=0.0, E_{3}=0.06\right.$, $\left.\mu_{1}=0.4\right)$

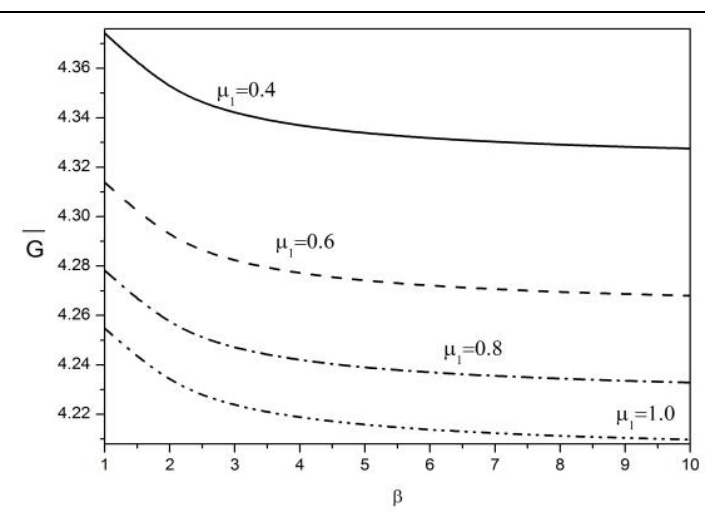

Fig. 13 : Effect of $\mu_{1}$ on $\bar{G}$ with $\beta$ $\left(\varepsilon=0.2, \alpha=0.5, E_{1}=0.1, E_{2}=4.0\right.$, $E_{3}=0.0, M=10$ )

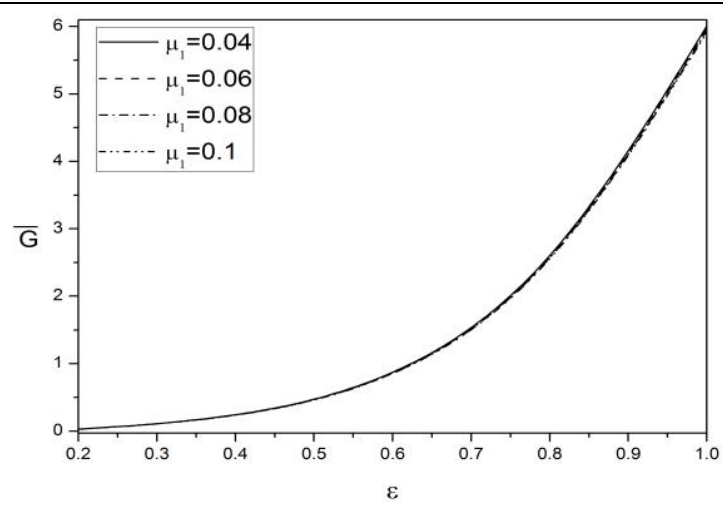

Fig. 14 : Effect of $\mu_{1}$ on $\bar{G}\left(\beta=5, \alpha=0.5, E_{1}=0.1, E_{2}=0.0, E_{3}=0.06, M=10\right)$ 